\title{
Letter to the Editor: New Approaches to Regulatory Innovation Emerging During the Crucible of COVID-19
}

\author{
Timothé Ménard, PharmD ${ }^{1}$ [
}

Received: 30 November 2020 / Accepted: 17 March 2021 / Published online: 22 March 2021

(c) The Drug Information Association, Inc 2021

\section{Dear Editor,}

I read with great interest the article by Wegner (New Approaches to Regulatory Innovation Emerging During the Crucible of COVID-19). The author examined options for companies to stay competitive while remaining compliant with agency regulations and guidance. These options focused on new ways of working (i.e. more flexible work model), on expanding the use of decentralized/hybrid trials, and on capitalizing on the use of real-world evidence (RWE) and cloud-based data systems [1].

We believe that other important considerations contributing to regulatory innovation in clinical drug development should be highlighted. For example, new ways of delivering quality assurance (QA) for clinical trials, by leveraging advanced analytics, had been developed in the recent years [2]. Other publications also emphasized the need to perform QA differently to address challenges posed by the pandemic and changing quality "to prevention rather than correction, critical thinking vs. editing, root cause analysis vs. symptom identification, systemic vs. isolated errors, partnering vs. policing, and applying solutions vs. fixing individual issues" [3].

Robust QA programs are required during the execution of clinical trials, to ensure patient safety and the integrity of the data that will be filed for regulatory approval [4]. Traditional clinical QA heavily relies on audits (or other retrospective investigations) to detect sites, studies or processes with quality issues. The increasing number of clinical trials and the growing complexity of study designs make it challenging to detect and identify clinical quality issues timely. Late detection of quality issues can lead to delayed filing, delayed approval and delayed patient access to innovative therapies [2]. Furthermore, the restrictions due the

Timothé Ménard

timothe.menard@roche.com

1 F. Hoffmann-La Roche, Basel, Switzerland pandemic (e.g. travel bans, lockdowns) forbade the possibility of on-site investigator QA audits. This constraint became an opportunity for sponsors to further apply their analytics solutions to perform quality oversight fully remotely. We recently published a case-study on how QA activities had been conducted for a phase III trial designed to evaluate the repositioning of tocilizumab to treat COVID-19 pneumonia [5]. There were several advantages of remote and analyticsdriven QA: ensuring business continuity, real-time detection and fast resolution of quality issues. This approach has the potential to reduce the need for on-site audits and thereby shift the focus away from source data validation and verification towards pre-identified, higher risk areas.

To transition from the experimentation phase that happened during the pandemic to the implementation of routine, remote and analytics-driven QA operations, sponsors and agencies will have to continue to collaborate and address challenges such as: fit-for-purpose Information Technology (IT) infrastructures, automation, cross-company QA data sharing, QA staff data literacy and models validation $[2,5]$. While the COVID-19 pandemic accelerated the adoption of new ways of working and pushed innovation further in the regulatory space, it also brought new rationales for a change in the QA paradigm, i.e. where advanced analytics can help conducting QA activities remotely, detecting and mitigating issues faster, and ultimately accelerate approval and patient access of innovative drugs.

\section{Funding}

No funding was provided for writing this letter.

\section{Declaration}

\section{Conflict of interest}

Timothé Ménard was employed by F.Hoffmann-La Roche (Roche) at the time this letter was completed. 


\section{References}

1. Wegner M. New approaches to regulatory innovation emerging during the crucible of COVID-19. Ther Innov Regul Sci. 2020. https://doi.org/10.1007/s43441-020-00239-8.

2. Koneswarakantha B, et al. Harnessing the power of quality assurance data: can we use statistical modeling for quality risk assessment of clinical trials? Ther Innov Regul Sci. 2020;54:1227-35. https://doi.org/10.1007/s43441-020-00147-x.

3. Alsumidaie M. Clinical Trial Quality Concerns Arise Amidst COVID. Applied Clinical Trials; 2020. https://www.appliedcli nicaltrialsonline.com/view/clinical-trial-quality-concerns-ariseamidst-covid. Accessed 30 Nov 2020.

4. Guideline for Good Clinical Practices - International Conference of Harmonization. https://database.ich.org/sites/default/files/E6_ R2_Addendum.pdf. Accessed 30 Nov 2020.

5. Menard $\mathrm{T}$, et al. Leveraging analytics to assure quality during the Covid-19 pandemic: the COVACTA clinical study example. Contemp Clin Trials Commun. 2020;20:100662. https://doi.org/ 10.1016/j.conctc.2020.100662. 